\title{
DIELECTRIC BEHAVIOUR, COMPLEX IMPEDANCE SPECTROSCOPY AND MAGNETOELECTRIC EFFECT IN LSMO-BT COMPOSITES
}

\author{
S. S. VEER \\ Department of physics, Arts, Science and Commerce College, Indapur, Pune, 413106, India \\ veershivaji1@gmail.com \\ S. V. KULKARNI \\ School of Physical Sciences, Solapur University, Solapur, 413 255, Pune, 413106, India. \\ suhas71@yahoo.com \\ S. D. CHAKANE \\ Department of Physics, Arts, Science and Commerce College, Indapur, Pune, 413106, India \\ schakane@gmail.com \\ P. B. JOSHI \\ School of Physical Sciences, Solapur University, Solapur, 413 255, India. \\ drpbjoshi@rediffmail.com
}

\begin{abstract}
The paper deals with Dielectric Behaviour, Complex Impedance Spectroscopy and Magnetoelectric effect in $\mathrm{La}_{0.7} \mathrm{Sr}_{0.3} \mathrm{MnO}_{3}(\mathrm{LSMO})$ and $\mathrm{BaTiO}_{3}(\mathrm{BT})$ Composites. The LSMO and BT are synthesized by hydroxide co-precipitation route. The nanocomposites are prepared by two series $x \mathrm{LSMO}+(1-x) \mathrm{BT}+2$ wt $\% \mathrm{Bi}_{2} \mathrm{O}_{3}$, for $x=0.05,0.1,0.15$ and $0.2 \ldots \ldots$ series 1. $x \mathrm{LSMO}+(1-x) \mathrm{BT}+3 \mathrm{wt} \% \mathrm{Bi}_{2} \mathrm{O}_{3}$, for $x=0.05,0.1,0.15$ and $0.2 \ldots \ldots$ series 2 . and sintered at $\mathrm{T}_{\mathrm{s}}=1000^{\circ} \mathrm{C}$ and $1080^{\circ} \mathrm{C}$. The composites are termed as LSMO-BT. The paper reports the crystal structure, micro-structural analysis, dielectric constant, complex impedance spectroscopy and magnetoelectric properties of LSMO-BT composites. It has been observed that the $\varepsilon_{\mathrm{r}}$ passes through a broad maximum at $\mathrm{T} \sim 124^{0} \mathrm{C}$, which is nearly the ferroelectric transition temperature of BT. Both $\varepsilon_{\mathrm{r}}$ and $\varepsilon_{\mathrm{rmax}}$ increases as $\mathrm{x}$ increases from 0.05 to 0.2 . The $\varepsilon_{\mathrm{r}}{ }^{\prime}$ and $\varepsilon_{\mathrm{r}}{ }^{\prime \prime}$ ' decreases sharply for all the compositions at low frequencies. This feature is typical of the presence of interfacial polarization. The variation of $4 \pi \mathrm{Ms}$ is almost linear with $\mathrm{x}$, the $4 \pi \mathrm{Ms}$ increases with $\mathrm{x}$ as well as sintering aid. The $4 \pi \mathrm{Ms}$ of respective compositions reduce as the sintering temperature is increased. The value of $\alpha$ increases for increase in sintering temperature as well as increase in wt $\%$ of the sintering aid. The magnitude of $\alpha$ is in the range as reported earlier for composites based on BT.
\end{abstract}

Keywords: Composites; Hydroxide co-precipitation; Dielectric constant; Magnetoelectric effect.

\section{Introduction}

The Ferroic materials have regained a renewed interest in the recent years; owing to the useful magnetoelectric susceptibility $\chi^{\mathrm{ME}}$ exhibited by the ferrite-Pb (TiZr) $\mathrm{O}_{3}(\mathrm{PZT})$, ferrite- $\mathrm{BaTiO}_{3}$ (BT) composites and multilayer laminates. In general the $\chi^{\mathrm{ME}}$ is large for the multilayers as compared to the composite compounds, ${ }^{1,2}$ and 3 .

The manganites are potential candidates to form ME composites for two reasons. 1) That they posses high value of magnetostriction and 2) The metallic conductivity of $\mathrm{La}_{0.7} \mathrm{Sr}_{0.3} \mathrm{MnO}_{3}$ (LSMO) at room temperature, which eliminates need of any foreign 
electrode at piezoelectric-Magnetostrictive interface ${ }^{1,2}$. The ME composites are expected to be mechanically sturdy as compared to the laminated composites ${ }^{4}$. Therefore we have opted to investigate the ME properties on particulate composites of LSMO-BT. To have well dispersed particles of LSMO and BT nanopowders of LSMO and BT are used as starting materials. Here, the hydroxide co-precipitation route is used for synthesis of LSMO and BT nanopowders $5,6,7$.

It has been reported that the use of sintering aid allows formation of dense ceramic bodies at relatively low sintering temperature ${ }^{8}$. The need of low sintering temperature is prompted from the observation of occurrence of impurity phases of LSMO and PZT for sintering above $1350 \mathrm{~K}^{2}$. The paper reports structural investigations on LSMO, BT nanopowders and composites using XRD spectra. The ac and dc conductivity, dielectric constant, complex impedance and magnetoelectric coefficient are investigated in light of finding a useful magnetoelectric material.

\section{Experimental}

Hydroxide co-precipitation route has been used for synthesis of LSMO and BT nanopowders.

\section{Synthesis of LSMO}

$\mathrm{La}\left(\mathrm{NO}_{3}\right)_{3} .6 \mathrm{H} 2 \mathrm{O}, \mathrm{Sr}\left(\mathrm{NO}_{3}\right)_{2}, \mathrm{MnCl}_{2} .4 \mathrm{H}_{2} \mathrm{O}, \mathrm{KMnO}_{4}$ of $\mathrm{AR}$ grade are used as precursors for synthesis of LSMO by hydroxide co-precipitation route. The weight proportions of $\mathrm{MnCl}_{2} \cdot 4 \mathrm{H}_{2} \mathrm{O}$ and $\mathrm{KMnO}_{4}$ is selected to be $3.56: 1$ so that the valencies of the hydroxide residues of $\mathrm{Mn}$ in state $3+$ and $4+$ would be $7: 3^{7}$. The precursors are dissolved in distilled water to form nearly $40 \mathrm{mM}$ solutions of the constituents. The $\mathrm{NH}_{4} \mathrm{OH}$ and $\mathrm{KOH}$ were used as mineralizers. The precipitates were thoroughly washed in distilled water keeping alkaline medium using $\mathrm{NH}_{4} \mathrm{OH}$ and dried under IR lamp. The dried precipitate is subjected to pre and final sintering process at $1223 \mathrm{~K}$ and $1473 \mathrm{~K}$, based on the analysis of TGA, DTG data on the hydroxide precipitate. This process has lead to the formation of LSMO particles of nearly $35 \mathrm{~nm}$, as discussed in the next section ${ }^{5}$.

\section{Synthesis of BT}

High purity $(>99.9 \%)$ Barium nitrate $\left[\mathrm{Ba}\left(\mathrm{NO}_{3}\right)_{2}\right]$ and Pottassium Titanium Oxalate $\left[\mathrm{K}_{2} \mathrm{TiO}\left(\mathrm{C}_{2} \mathrm{O}_{4}\right)_{2} \cdot 2 \mathrm{H}_{2} \mathrm{O}\right]$ are used as precursors. The $\mathrm{KOH}$ has been employed as a mineralizer. For complete precipitation of $\mathrm{Ba}(\mathrm{OH})_{2}$ and $\mathrm{Ti} \mathrm{O}(\mathrm{OH})_{2}$, the molar ratio of $\mathrm{KOH}$ to $(\mathrm{BaTi})$ of 1.6 has been used, based on the earlier reports ${ }^{9}$. It has been observed that the $\mathrm{Ba}(\mathrm{OH})_{2}$ is fractionally soluble in water but insoluble in alkaline medium ${ }^{10}$. Therefore the precipitates are washed in dilute $\mathrm{NH}_{4} \mathrm{OH}$ solution with $\mathrm{p}^{\mathrm{H}} \sim 8$. The remaining procedure of co-precipitation is similar to that of LSMO. The precipitates are subjected to TG analysis to examine the validity of proposed precipitation reactions and to determine the minimum required sintering temperature. Considering TG analysis the precipitates of $\mathrm{Ba}(\mathrm{OH})_{2}$ and $\mathrm{TiO}(\mathrm{OH})_{2}$ are subjected to the pre sintering process at 1473 $\mathrm{K}$ and the final sintering has been carried out at $1553 \mathrm{~K}$ for $4 \mathrm{hrs}$.

The nano powders of LSMO and BT are used to form composites using formulae of series 1 and series 2 as above. The $\mathrm{Bi}_{2} \mathrm{O}_{3}$ is used as sintering aid to lower the temperature of sintering and achieve higher levels of densification ${ }^{8}$. The composites are formed after through mixing of LSMO, BT and $\mathrm{Bi}_{2} \mathrm{O}_{3}$ powders in acetone medium. The composites are formed in pellet shape of $1 \mathrm{~cm}$ in diameter and are sintered at $1273 \mathrm{~K}$ for 8 hours. 
These pellets are used for further process of investigation. The HP4284A LCR-Q meter is used for the purpose of the measurement of dielectric constant, ac conductivity and complex impedance spectra. The custom built setups are used for the measurement of dc conductivity and magneto-electric effect.

\section{Results and Discussion}

\section{XRD and particle size}

The individual powders of LSMO and BT are subjected to the X-ray analysis after the pre sintering and final sintering processes. The X-ray spectra of LSMO are observed to be in accordance with the standard data (ICDD card \# 89-4461). The observed peak widths of $\mathrm{X}$-ray spectra are used to determine the particle size using Williamson Hall method. The analysis shows that LSMO posses particle size of nearly $35 \mathrm{~nm}$. The XRD spectrum of BT is observed to reproduce the JCPD data and is in confirmation with the recent observation of XRD on hydrothermally synthesized BT powder ${ }^{11}$. The particle size estimated using Williamson Hall method is observed to be nearly $120 \mathrm{~nm}$. Therefore it appears that the hydroxide co-precipitation route could be used to produce nano scale ceramics. The XRD spectra on pellets of the composites are observed to show the reflections corresponding to both the LSMO and BT phases and confirmed formation of the desired composite form.

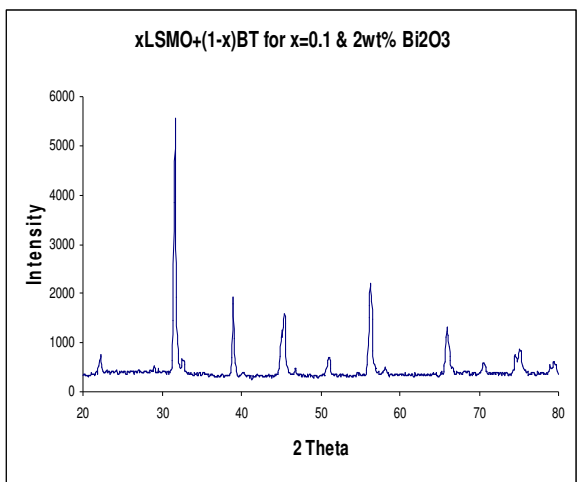

Fig. 1 XRD Spectra of S1for $\mathrm{x}=0.1$.

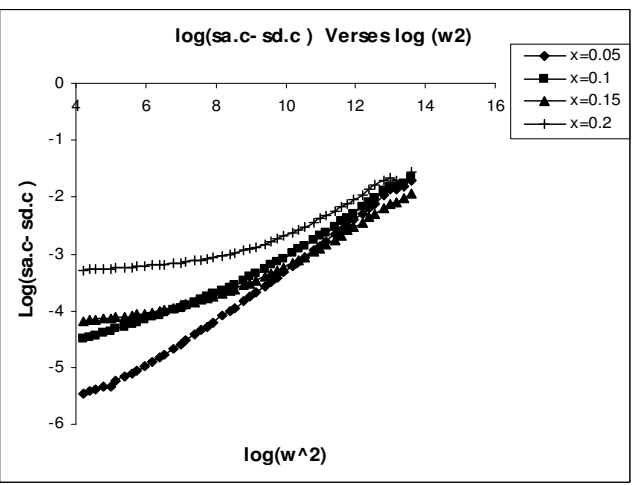

Fig. $2 \log \left(\sigma_{\mathrm{ac}}-\sigma_{\mathrm{dc}}\right)$ Vs $\log \omega^{2}$ of series 2 .

Fig. 1 shows XRD spectra for $\mathrm{x}=0.1$ of series 1 . Here from fig. 1 it could be seen that the reflections at $\theta=31.5,38.9,44.9,45.4,56.3$ of BT and at $\theta=32.6,38.3,46.9$ of LSMO are well separated from each other. Further no additional peak corresponding to any impurity phase is seen in fig. 1. Thus, formation of the composite phase is confirmed without occurrence of any impurity phase within detectable range of XRD spectra.

The resistance of the composites is expected to be due to the presence of BT and therefore may indicate frequency variation characteristics of small polarons. The resistivity of the BT and other ferroelectric compounds is known to be due to the formation of small polaron ${ }^{12}$. To determine whether the conduction is due to small polarons, the ac conductivity $\sigma_{\mathrm{ac}}$ and complex impedance is measured for series 1 and series 2 . For the conduction due to small polarons the following relations is expected to hold $\operatorname{good}^{13}$.

$$
\sigma_{\mathrm{ac}}=\sigma_{\mathrm{dc}}+\omega^{2} \tau^{2} /\left(1+\omega^{2} \tau^{2}\right)
$$


Where $\tau$ is the staying time of a polaron. For $\omega$ up to $1 \mathrm{MHz}$, the $\omega^{2} \tau^{2}$ is $<<1$ and the equation (2) becomes

$$
\sigma_{\mathrm{ac}}-\sigma_{\mathrm{dc}}=\omega^{2} \tau^{2}
$$

Fig. 2 shows variation of $\log \left(\sigma_{\mathrm{ac}}-\sigma_{\mathrm{dc}}\right)$ as a function of $\log \omega^{2}$ for series 1 .The almost linear variation of $\log \left(\sigma_{\mathrm{ac}}-\sigma_{\mathrm{dc}}\right)$ with $\log \omega^{2}$, confirms that the conduction in these compositions is due to small polarons. The behavior of series 2 is similar to that of 1 . Fig. 3 shows the variation of the dielectric constant $\varepsilon_{\mathrm{r}}$, at excitation frequency $\mathrm{f}=1 \mathrm{KHz}$, as a function of $\mathrm{T}$ for series 1 . The overall behavior for series 2 also is similar to these observations except for the magnitude of $\varepsilon_{\mathrm{r}}$. It is observed that the $\varepsilon_{\mathrm{r}}, \varepsilon_{\mathrm{rmax}}$ (the maximum value of $\varepsilon_{\mathrm{r}}$ at $\mathrm{T}=\mathrm{T}_{\mathrm{c}}$ ) and $\mathrm{Q}$ for series 2 are lower as compared that of series 1 . Now, the features common for series 1 and series 2 could be summarized as (i) the $\varepsilon_{\mathrm{r}}$ passes through diffused phase transistion (DPT) at $\mathrm{T} \sim 387 \mathrm{~K}$, (ii) for frequencies less than 100 $\mathrm{KHz}$, the $\varepsilon_{\mathrm{r}}$ shows possibility of another DPT for $\mathrm{T} \sim 300 \mathrm{~K}$ or less, but for $\mathrm{f}=100 \mathrm{KHz}$ and $1 \mathrm{MHz}, \varepsilon_{\mathrm{r}}$ do not show any significant DPT behavior in this temperature range as shown in fig. 3 and (iii) The $\varepsilon_{\text {rmax }}$ is observed to increase with increasing $\mathrm{x}$, while the $\mathrm{Q}$ reduces with increasing $\mathrm{x}$.

The frequency variation of $\varepsilon_{\mathrm{r}}$ shows a characteristic behavior indicating a presence of interfacial/space charge polarization. Fig. 4 shows variation of $\varepsilon_{\mathrm{r}}$ as a function of $\mathrm{T}$ for varying f for $x=0.15$ of series 2 . From fig. 4 it could be seen that the $\varepsilon_{\mathrm{r}}$ and $\varepsilon_{\mathrm{rmax}}$ reduce sharply with increasing $x$. The behaviour for other compositions is observed similar to the one shown in fig. 4.

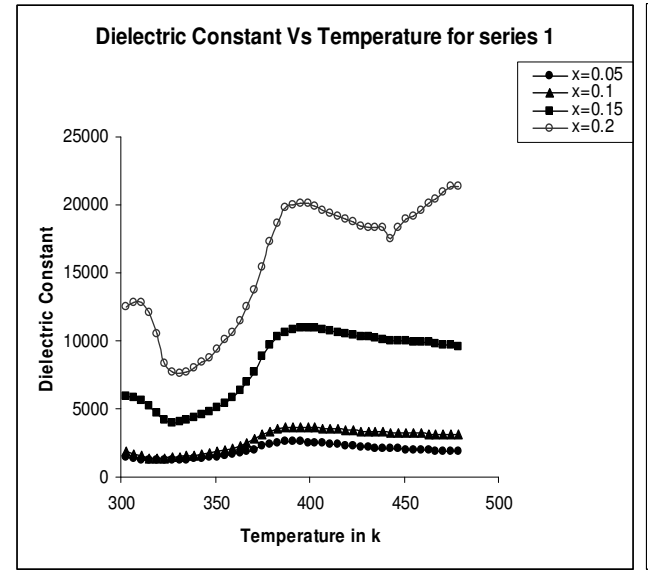

Fig. $3 \varepsilon_{\mathrm{r}} \mathrm{Vs} \mathrm{T}$ of series 1 at $\mathrm{f}=1 \mathrm{KHz}$.

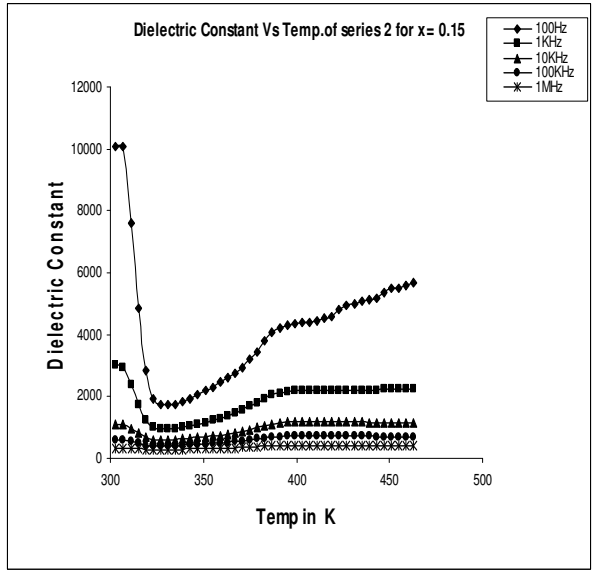

Fig. $4 \varepsilon_{\mathrm{r}}$ Vs $\mathrm{T}$ of series 2 for $\mathrm{x}=0.15$.

These observations of $\varepsilon_{\mathrm{r}}$ could be understood by assuming two contributions to the $\varepsilon_{\mathrm{r}}$, one due to the parent BT particles and other due to interfacial/space charge polarization at grain boundaries of LSMO and BT occurring because of very large difference in the conductivities of these two sub-systems.

The DPT at nearly $387 \mathrm{~K}$ would be because of presence of BT particles, while the behaviour of $\varepsilon_{\mathrm{r}}$ in the paraelectric region $(\mathrm{T}>387 \mathrm{~K})$ could be due to the presence of inter-grain interfacial/space charge polarization. It is observed that the interfacial/space charge polarization is significant at low frequencies and at high temperatures ${ }^{14}$. This may 
lead to very large values of $\varepsilon_{\mathrm{rmax}}$. From fig. 4 it is observed that the $\varepsilon_{\mathrm{r}}$ and $\varepsilon_{\mathrm{rmax}}$ are very large for $100 \mathrm{~Hz}$. Further the fig. 4 shows that $\varepsilon_{\mathrm{r}}$ and $\varepsilon_{\mathrm{rmax}}$ increase as $\mathrm{x}$ is increased. Both these features are out product of the inter grain-interfacial polarization. Similar effects are reported for the composites of $\left(\mathrm{Ba}_{0.5} \mathrm{Sr}_{0.5}\right) \mathrm{Nb}_{2} \mathrm{O}_{6}$ and ferrite coposites ${ }^{4}$. A DPT that is seen near room temperature is significant only at low frequencies ( $\mathrm{f}<10 \mathrm{KHz}$ ) and for $x>$ 0.15 . This feature could be associated to the diffusion of $\mathrm{Mn}$ ions at the grain boundaries of BT. This diffusion may cause grain-grain boundary effects in case of BT particles. Further, the BT is known to possess an orthorhombic to tetragonal phase transition in the vicinity of $280 \mathrm{~K}$, where the modified surface of BT may cause a change in the overall behavior of $\varepsilon_{\mathrm{r}}$ due to this phase transition. The earlier observations of LSMO-PZT composites have shown occurrence of impurity phases for sintering above $1350 \mathrm{~K}^{2}$. The similar phenomenon may cause a diffusion of the Mn or Ti ions in BT and LSMO matrices, though no impurity phase occurs at this sintering temperature $(1273 \mathrm{~K})$. To understand this feature in details, low temperature measurements of $\varepsilon_{\mathrm{r}}$ and saturation magnetization of composites would be required.

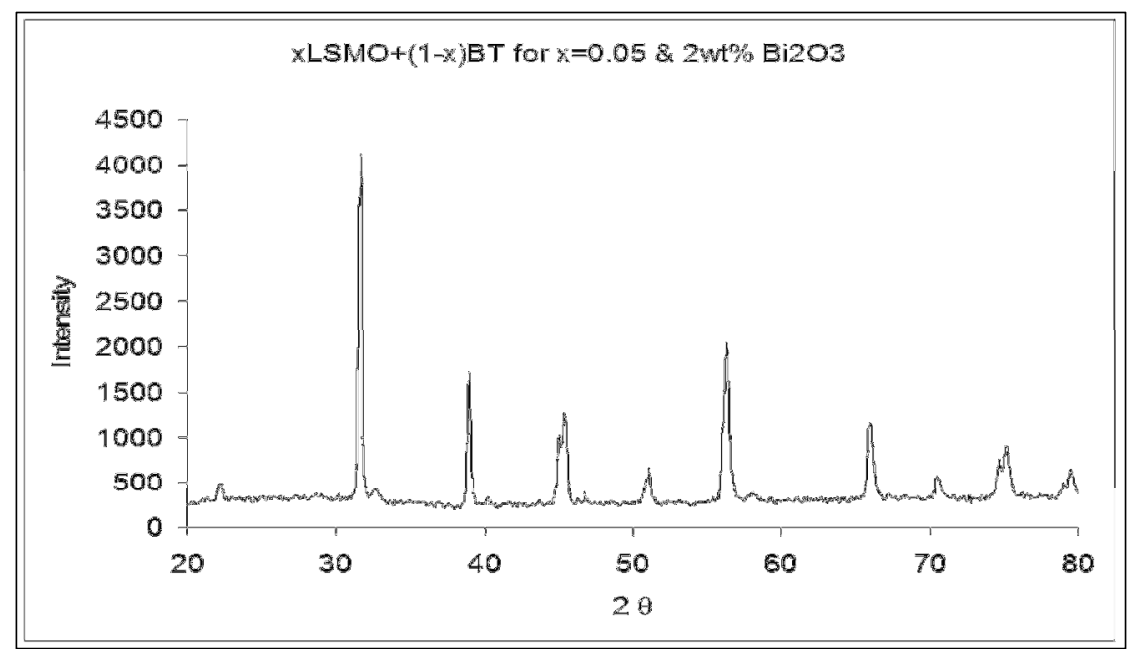

Fig. 5 XRD of $x L S M O+(1-x) B T$ for $x=0.5$ series 1

Fig. 5 shows the XRD of LSMO-BT composite for $\mathrm{x}=0.5$ series 1 . It shows $\mathrm{I} / \mathrm{I}_{0}$ increases from 0.05 t0 0.2 for both the series. XRD also shows the LSMO and BT phases are separate. For the measurements of longitudinal magnetoelectric coefficient $\alpha_{33}$, the samples are polled at an electric field of $1.5 \mathrm{KV} / \mathrm{cm}$ for 8 hours, which sufficient to poll the BT particles. The $\alpha_{33}$ is measured at $800 \mathrm{~Hz}$, with zero dc bias field, using a laboratory scale setup ${ }^{1}$. The Fig. 6 and Fig. 7 show variation of $\alpha$ Vs $X$ for series 1 and 2 , respectively. It has been observed that the variation of $\alpha \square$ with $\mathrm{x}$ and Ts show similar relationship as reported earlier for CZFO - PZT and NZFO - PZT composites. The observed magnitudes of $\alpha_{33}$ are within the range of values reported earlier for composites of ferrite-BT and LSMO-PZT ${ }^{1,2}$. Further investigations on the magnetoelectric coefficient are in progress using lock-in analyzer and FET preamplifier circuit. 


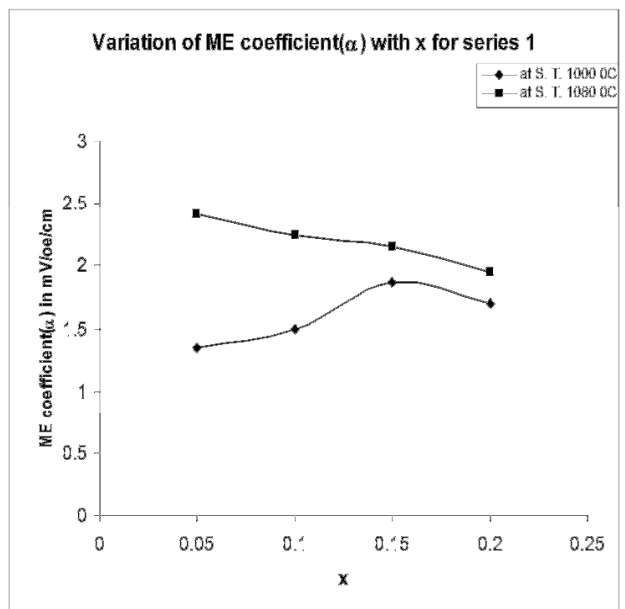

Fig. $6 \alpha \mathrm{Vs} \mathrm{X}$ for series 1

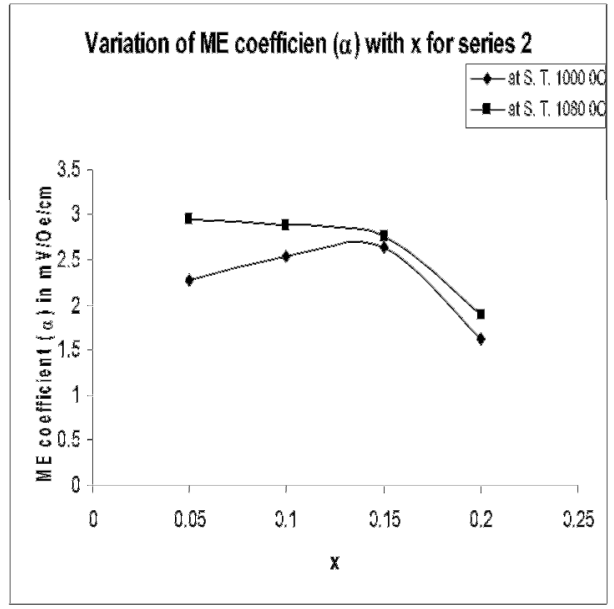

Fig.7 $\alpha \mathrm{Vs} \mathrm{X}$ for series 2

\section{Conclusions}

Hydroxide co-precipitation route has been successfully used for synthesis of nanocrystalline LSMO and BT powders. To form dense composites of LSMO-BT at lower temperatures $(1273 \mathrm{~K}), \mathrm{Bi}_{2} \mathrm{O}_{3}$ could be used as a sintering aid. The preliminary investigations on $\sigma_{\mathrm{dc}}, \varepsilon_{\mathrm{r}}$ and $\alpha_{33}$ indicate that LSMO-BT composites will also posses a useful figure of merit for the device applications.

\section{References}

1. G Srinivasan, E T Rasmussen, J Galleges \& R Hayes, Phy.Rev.B. 67,(2003)144418.

2. G Srinivasan, E T Rasmussen J Galleges and R Hayes, Phy.Rev.B. 64,(2001)214408.

3. Junyi Zhai, Ning Cai,Zhan Shi, Yuanhua Lin and C-Wen Nan, J.phys. D: Appl. Phys. 37, (2004)823.

4. Y J Li, X M Chen, Y Q Lin \& Y H Tang, Journal of the European Ceramic Society, 26(2006) 2839.

5. D J Salunkhe, Y D Kolekar, S B Kulkarni and P B Joshi, proceedings of International Conference on Nanomaterials, Nano 2005 , Vol I,149.

6. Gang Xu, Wenjian Weng Jianxi Yao, Piyi Du \&Gaorong Han, Microelectronic Engineering 66, (2003) 568.

7. S A Mirji, Y B Khollam, S B Deshpande, H S Potdar, R N Bhate, S R Sainkar, S K Date, Materials letters 58 ,(2004) 837.

8. Amitava Chakraborty \& Himadri S Maiti, Ceramic International, 25(1999) 115.

9. Lee S K, Park T J, Choi G J, Koo K K \& Sang Woo Kim, Materials Chemistry and Physics, 82(2003) 742.

10. John A Dean, Lenzes Handbook of Chemistry, $13^{\text {th }}$ edition Mc-Graw-Hill Book Company, pp 4-26, 4-67.

11. Huarui Xu, Lian Gao, Jinkun Guo, Journal of the European Ceramic Society, 22 (2002) 1163.

12. K K Patankar, V L Mathe, A N Patil, S A Patil, S D Lotke, Y D Kolekar and P B Joshi, Journal of Electroceramics 6:2, (2001) 115. 
13. D Alder, J Feinleib , Phys. Rev .B2 , 3112 (1970) 3112.

14. Nanda Goswami M L, Mahapatra P K\& Choudhary R N P, Indian Journal of Pure \& Applied Physics, 35(1997) 743.

15. Lakshman Pandey, Rajesh Katare, Om Parkash and Devendra Kumar, Bulletin of Materials Science, 20, 7 (1997) 933. 